Gazi University
Journal of Science
PART A: ENGINEERING AND INNOVATION
$\underline{h t t p: / / d e r g i p a r k . g o v . t r / g u j s a}$

\title{
Formulae to Fubini Type Numbers emerge from Application of $\boldsymbol{p}$-adic Integrals
}

\author{
Neslihan KILAR $^{1 *(D)}$, Yilmaz SIMSEK ${ }^{2}(\mathbb{D})$ \\ ${ }^{I}$ Niğde Ömer Halisdemir University, Bor Vocational School, Department of Computer Technologies, Niğde TR-51700 Turkey \\ ${ }^{2}$ Akdeniz University, Faculty of Science, Department of Mathematics, Antalya TR-07058, Turkey
}

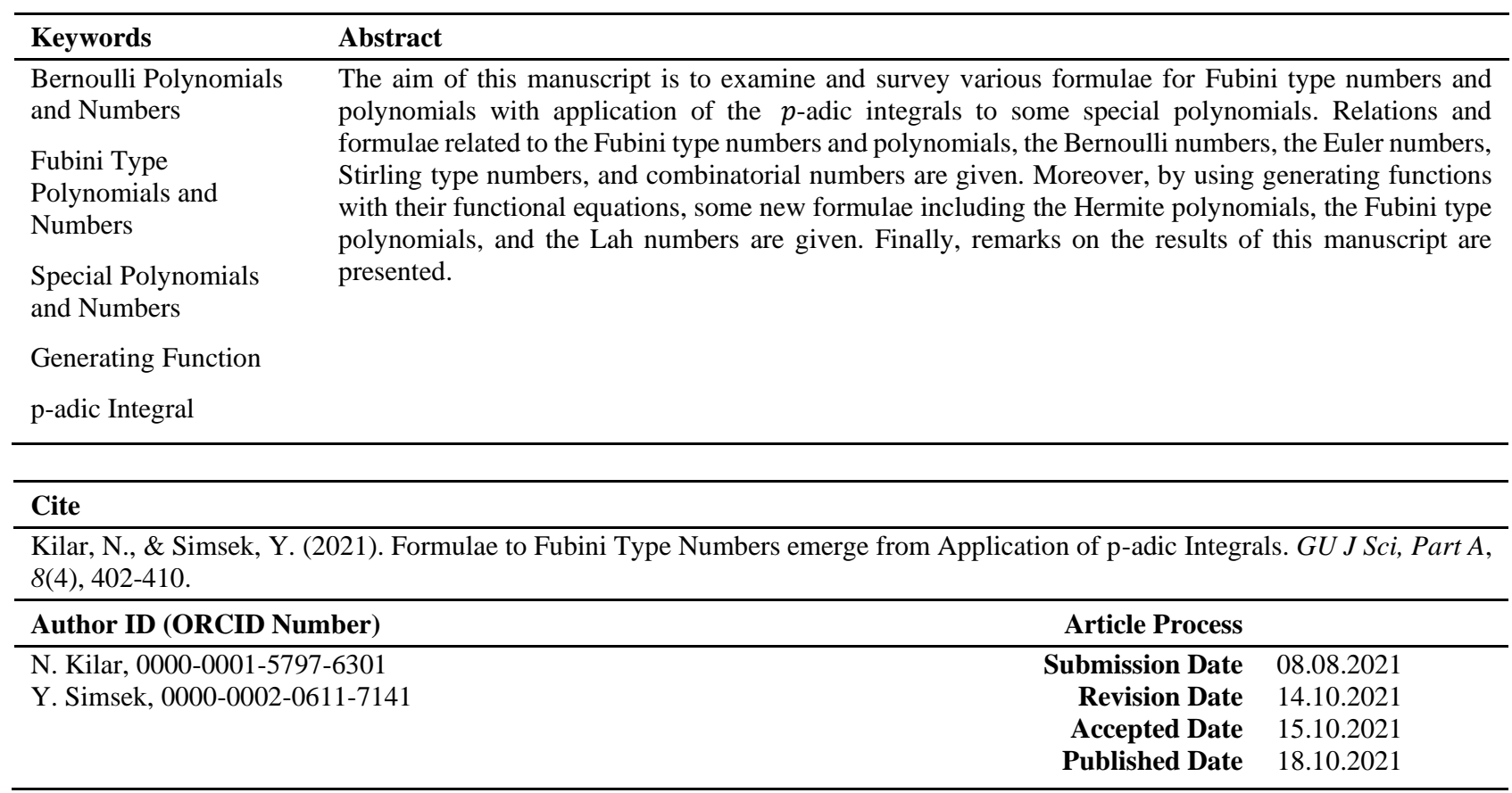

\section{INTRODUCTION}

In “The On-Line Encyclopedia of Integer Sequences” (OEIS, 2021) (https://oeis.org/A000670), it is well-know that the Fubini numbers are related to the number of preferential arrangements of $n$ labeled elements, and also the number of ordered partitions of $[n]$. The Fubini numbers are also called the ordered Bell numbers. Later, by Comtet (1974), these numbers were also called the Fubini numbers.

Kilar \& Simsek (2017) modified these numbers and defined new generalized Fubini type numbers and polynomials. They also gave very different and interesting applications of these numbers and polynomials with aid of the generating functions and their functional equations. Recently, it is known that these type numbers and polynomials have been studied by many mathematicians using different methods and fields (Belbachir et al., 2011; Kilar, 2017; 2021; Kilar \& Simsek, 2017; 2019a,b; Kim et al., 2018; Srivastava \& Kızılateş, 2019).

Some definitions and notations connected with special polynomials and numbers and their generating functions are presented as follows:

Let $\mathbb{N}=\{1,2,3, \ldots\}$ and $\mathbb{N} \cup\{0\}=\mathbb{N}_{0}$. Let $\mathbb{Z}=\mathbb{N} \cup\{0,-1,-2,-3, \ldots\}$. Let $\mathbb{C}$ indicate the set of complex numbers and $\mathbb{Z}_{p}$ indicate the set of $p$-adic integers.

$$
\left(\begin{array}{l}
u \\
c
\end{array}\right)=\frac{u(u-1) \ldots(u-c+1)}{c !}=\frac{(u)_{c}}{c !}
$$


where $c \in \mathbb{N}, u \in \mathbb{C}$ and $(u)_{0}=1$ (Belbachir et al., 2011;-; Srivastava \& Choi, 2012).

Generating function of the classical Bernoulli polynomials is given by

$$
\frac{z}{e^{z}-1} e^{t z}=\sum_{m=0}^{\infty} B_{m}(t) \frac{z^{m}}{m !},
$$

where $|z|<2 \pi$ (Comtet, 1974;-; Srivastava \& Choi, 2012).

Setting $t=0$ in (1), we see that

$$
B_{m}(0)=B_{m}
$$

denoted the classical Bernoulli numbers (Comtet, 1974;-; Srivastava \& Choi, 2012).

Generating function of the classical Euler polynomials is given by

$$
\frac{2}{e^{z}+1} e^{t z}=\sum_{m=0}^{\infty} E_{m}(t) \frac{z^{m}}{m !},
$$

where $|z|<\pi$ (Comtet, 1974;-; Srivastava \& Choi, 2012).

Setting $t=0$ in (2), we observe that

$$
E_{m}(0)=E_{m}
$$

denoted the classical Euler numbers (Comtet, 1974;-; Srivastava \& Choi, 2012).

Generating function of the Hermite polynomials is given by

$$
G_{H}(z, \mathrm{t})=e^{2 t z-z^{2}}=\sum_{m=0}^{\infty} H_{m}(t) \frac{z^{m}}{\mathrm{~m} !},
$$

(Rainville, 1960).

Using equation (3), we have

$$
t^{n}=\sum_{k=0}^{\left[\frac{n}{2}\right]} \frac{n ! H_{n-2 k}(t)}{2^{n} k !(n-2 k) !}
$$

(Rainville, 1960).

Let $c \in \mathbb{N}_{0}$ and $a \in \mathbb{C}$. Generating function of Stirling type numbers is given by

$$
\frac{\left(a e^{z}-1\right)^{c}}{c !}=\sum_{d=0}^{\infty} S_{2}(d, c ; a) \frac{z^{d}}{d !}
$$

(Simsek, 2013; 2019).

Generating function of the Stirling numbers of the second kind is given by 


$$
\frac{\left(e^{z}-1\right)^{c}}{c !}=\sum_{d=0}^{\infty} S_{2}(d, c) \frac{z^{d}}{d !}
$$

and

$$
t^{c}=\sum_{m=0}^{c} S_{2}(c, m)(t)_{m}
$$

(Comtet, 1974;-; Srivastava \& Choi, 2012).

Substituting $a=1$ into (5), we have

$$
S_{2}(d, c ; 1)=S_{2}(d, c)
$$

For $c>d$, one has

$$
S_{2}(d, c)=0
$$

(Comtet, 1974;-; Srivastava \& Choi, 2012).

An explicit formula for the Lah numbers is given by

$$
L(c, s)=\frac{(-1)^{c} c !\left(\begin{array}{l}
c-1 \\
s-1
\end{array}\right)}{s !}
$$

where $c \geq s \geq 1, L(0,0)=1$ and $L(c, s)=0$ for all $s>c$ (Riordan, 1958; Comtet, 1974). Here note that this numbers are so called signed Lah numbers.

By the aid of the equation (8), we have

$$
(r)_{c}=\sum_{s=0}^{c} L(c, s)(-r)_{s}
$$

(Riordan, 1958 (p.43); Comtet, 1974 (p.156)).

The Daehee numbers are given by

$$
\frac{\ln (1+z)}{\mathrm{z}}=\sum_{m=0}^{\infty} D_{m} \frac{z^{m}}{m !}
$$

(Kim \& Kim, 2013; 2018; Simsek, 2016; 2019).

By using (10), we get

$$
D_{v}=\frac{(-1)^{v} v !}{v+1}
$$

(Kim \& Kim, 2013; 2018; Simsek, 2016; 2019).

The Changhee numbers $C h_{m}$ are given by 


$$
\frac{2}{\mathrm{z}+2}=\sum_{m=0}^{\infty} C h_{m} \frac{z^{m}}{\mathrm{~m} !}
$$

(Kim et al., 2013; Simsek, 2019). By using (11), we have

$$
C h_{v}=\frac{(-1)^{v} v !}{2^{v}}
$$

(Kim et al., 2013; Simsek, 2019).

Generating function of the Fubini type polynomials of order $c$ is given by

$$
G_{a}(z, t, c)=\frac{2^{c}}{\left(2-e^{z}\right)^{2 c}} e^{t z}=\sum_{m=0}^{\infty} a_{m}^{(c)}(t) \frac{z^{m}}{m !},
$$

where $|z|<\ln 2$ and $c \in \mathbb{N}_{0}$ (Kilar \& Simsek, 2017; see also Kilar, 2017; Kilar \& Simsek, 2019a,b).

Setting $t=0$ in (12), we have the Fubini type numbers of order $c$ :

$$
a_{m}^{(c)}(0)=a_{m}^{(c)}
$$

(Kilar \& Simsek, 2017; see also Kilar, 2017; Kilar \& Simsek, 2019a,b).

Using (12), we get

$$
a_{d}^{(c)}(t)=\sum_{k=0}^{\mathrm{d}}\left(\begin{array}{l}
d \\
k
\end{array}\right) a_{k}^{(c)} t^{d-k}
$$

(Kilar \& Simsek, 2017; Kilar, 2017).

\subsection{Formulas for $\boldsymbol{p}$-adic Integrals and Some Special Numbers}

Here, we give some formulas including the $p$-adic integrals involving the Volkenborn integral and the $p$-adic Fermionic integral and special numbers. These formulas have many applications in physics and in engineering besides in mathematics.

Let $C^{1}\left(\mathbb{Z}_{p} \rightarrow K\right)$ denotes the set of the uniformly differential function $f$ on $\mathbb{Z}_{p}$.

The Volkenborn integral (or the bosonic $p$-adic integral) of the uniformly differential function $f$ on $\mathbb{Z}_{p}$ is given by

$$
\int_{\mathbb{Z}_{p}} f(x) d \mu_{1}(x)=\lim _{N \rightarrow \infty} \frac{1}{p^{N}} \sum_{x=0}^{p^{N}-1} f(x),
$$

where $f \in\left(\mathbb{Z}_{p} \rightarrow \mathrm{K}\right)$ and

$$
\mu_{1}(x)=\mu_{1}\left(x+p^{N} \mathbb{Z}_{p}\right)=p^{-N}
$$

(Schikhof, 1984; Kim, 2002a; 2005; Kim \& Kim, 2013; Simsek, 2019; 2021).

Using (14), the Bernoulli numbers $B_{m}$ is also given by 


$$
\int_{\mathbb{Z}_{p}} x^{m} d \mu_{1}(x)=B_{m}
$$

(Schikhof, 1984; Kim, 2002a; Kim \& Kim, 2013; Simsek, 2019).

Using (14), the Daehee numbers $D_{m}$ is also given by

$$
\int_{\mathbb{Z}_{p}}(x)_{m} d \mu_{1}(x)=D_{m}
$$

(Kim, 2002b; Kim \& Kim, 2013; Simsek, 2019).

Let $f \in\left(\mathbb{Z}_{p} \rightarrow \mathrm{K}\right)$. The $p$-adic Fermionic integral of the uniformly differential function $f$ on $\mathbb{Z}_{p}$ is given by

$$
\int_{\mathbb{Z}_{p}} f(x) d \mu_{-1}(x)=\lim _{N \rightarrow \infty} \sum_{x=0}^{p^{N}-1}(-1)^{x} f(x),
$$

Where

$$
\mu_{-1}(x)=(-1)^{x}
$$

(Kim, 2007; Simsek, 2019).

Using (17), the Euler numbers $E_{m}$ is also given by

$$
\int_{\mathbb{Z}_{p}} x^{m} d \mu_{-1}(x)=E_{m}
$$

(Kim, 2007; Simsek, 2019).

Using (17), the Changhee numbers $C h_{m}$ is also given by

$$
\int_{\mathbb{Z}_{p}}(x)_{m} d \mu_{-1}(x)=C h_{m}
$$

(Kim et al., 2013; Simsek, 2019).

\section{FORMULAE FOR FUBINI TYPE NUMBERS: APPROACH TO APPLICATION OF P-ADIC INTEGRALS}

By using the $p$-adic integrals and functional equations of the generating functions, we give some formulae and finite sums including the Fubini type polynomials and numbers of higher order, the Bernoulli numbers, the Euler numbers, the Lah numbers, the Stirling type numbers, combinatorial numbers, and also the Hermite polynomials.

For $v \in \mathbb{N}_{0}$, Kilar (2017; Corollary 4.2, p. 28) gave the following identity:

$$
x^{v}=(2 c) ! 2^{c} \sum_{r=0}^{v}\left(\begin{array}{l}
v \\
r
\end{array}\right) S_{2}\left(r, 2 c ; \frac{1}{2}\right) a_{v-r}^{(c)}(x) .
$$


By using (20) and (7), the following result is derived:

Corollary 2.1. Let $v \in \mathbb{N}_{0}$. Then we have

$$
\sum_{s=0}^{v} S_{2}(v, s)(x)_{s}=(2 c) ! 2^{c} \sum_{r=0}^{v}\left(\begin{array}{l}
v \\
r
\end{array}\right) S_{2}\left(r, 2 c ; \frac{1}{2}\right) a_{v-r}^{(c)}(x) .
$$

Combining (21) with (9), we derive the following relation involving the Lah numbers, the Stirling type numbers, and the Fubini type polynomials of higher order:

Theorem 2.2. Let $v \in \mathbb{N}_{0}$. Then we have

$$
\sum_{s=0}^{v} \sum_{d=0}^{s} S_{2}(v, s) L(s, d)(-x)_{d}=(2 c) ! 2^{c} \sum_{r=0}^{v}\left(\begin{array}{l}
v \\
r
\end{array}\right) S_{2}\left(r, 2 c ; \frac{1}{2}\right) a_{v-r}^{(c)}(x) .
$$

Theorem 2.3. Let $v \in \mathbb{N}_{0}$. Then we have

$$
a_{v}^{(c)}(2 x)=\sum_{r=0}^{\left[\frac{v}{2}\right]} \sum_{s=0}^{v-2 r}\left(\begin{array}{c}
v-2 r \\
s
\end{array}\right)\left(\begin{array}{c}
v \\
2 r
\end{array}\right) a_{s}^{(c)} H_{v-2 \mathrm{r}-s}(x) .
$$

Proof. Multiplying the function $\frac{2^{c}}{\left(2-e^{Z}\right)^{2 c}}$ on the both-sides of (3), after that using the resulting equation and (12), we obtain

$$
G_{a}(z, 2 x, c)=e^{z^{2}} G_{a}(z, 0, c) G_{H}(z, x)
$$

With the help of the above functional equation, we get

$$
\sum_{v=0}^{\infty} a_{v}^{(c)}(2 x) \frac{z^{v}}{v !}=\sum_{v=0}^{\infty} \frac{z^{2 v}}{v !} \sum_{v=0}^{\infty} a_{v}^{(c)} \frac{z^{v}}{v !} \sum_{v=0}^{\infty} H_{v}(x) \frac{z^{v}}{v !} .
$$

Thus

$$
\sum_{v=0}^{\infty} a_{v}^{(c)}(2 x) \frac{z^{v}}{v !}=\sum_{v=0}^{\infty} \sum_{r=0}^{\left[\frac{v}{2}\right]} \sum_{s=0}^{v-2 r}\left(\begin{array}{c}
v-2 r \\
s
\end{array}\right)\left(\begin{array}{c}
v \\
2 r
\end{array}\right) a_{s}^{(c)} H_{v-2 \mathrm{r}-s}(x) \frac{z^{v}}{v !}
$$

Therefore, we arrive at the desired result.

Applying the Volkenborn integral to (20), then make use of the final equation with (13) and (15), we obtain the following result:

Theorem 2.4. Let $v \in \mathbb{N}_{0}$. Then we have

$$
B_{v}=(2 c) ! 2^{c} \sum_{r=0}^{v}\left(\begin{array}{l}
v \\
r
\end{array}\right) S_{2}\left(r, 2 c ; \frac{1}{2}\right) \sum_{l=0}^{v-r}\left(\begin{array}{c}
v-r \\
l
\end{array}\right) a_{l}^{(c)} B_{v-r-l} .
$$

Applying the Volkenborn integral to (21), after that using the resulting equation with (15) and (16), we have the following result: 
Theorem 2.5. Let $v \in \mathbb{N}_{0}$. Then we have

$$
\sum_{s=0}^{v} S_{2}(v, s) D_{s}=(2 c) ! 2^{c} \sum_{r=0}^{v}\left(\begin{array}{l}
v \\
r
\end{array}\right) S_{2}\left(r, 2 c ; \frac{1}{2}\right) \sum_{l=0}^{v-r}\left(\begin{array}{c}
v-r \\
l
\end{array}\right) a_{l}^{(c)} B_{v-r-l}
$$

or, equivalently,

$$
\sum_{s=0}^{v}(-1)^{s} \frac{s ! S_{2}(v, s)}{s+1}=(2 c) ! 2^{c} \sum_{r=0}^{v}\left(\begin{array}{l}
v \\
r
\end{array}\right) S_{2}\left(r, 2 c ; \frac{1}{2}\right) \sum_{l=0}^{v-r}\left(\begin{array}{c}
v-r \\
l
\end{array}\right) a_{l}^{(c)} B_{v-r-l} .
$$

Remark 2.6. Combining (23) with (22), we get

$$
\sum_{s=0}^{v} S_{2}(v, s) D_{s}=B_{v}
$$

where $v \in \mathbb{N}_{0}$ (Kim \& Kim, 2013; Simsek, 2019).

Applying the $p$-adic Fermionic integral to (20), then using final equation and equations (13) and (18), we get Theorem 2.7 as follows.

Theorem 2.7. Let $v \in \mathbb{N}_{0}$. Then we have

$$
E_{v}=(2 c) ! 2^{c} \sum_{r=0}^{v}\left(\begin{array}{l}
v \\
r
\end{array}\right) S_{2}\left(r, 2 c ; \frac{1}{2}\right) \sum_{l=0}^{v-r}\left(\begin{array}{c}
v-r \\
l
\end{array}\right) a_{l}^{(c)} E_{v-r-l} .
$$

Applying the $p$-adic Fermionic integral to (21), then make use of the final equation with (18) and (19), we derive Theorem 2.8 below.

Theorem 2.8. Let $v \in \mathbb{N}_{0}$. Then we have

$$
\sum_{s=0}^{v} S_{2}(v, s) C h_{s}=(2 c) ! 2^{c} \sum_{r=0}^{v}\left(\begin{array}{l}
v \\
r
\end{array}\right) S_{2}\left(r, 2 c ; \frac{1}{2}\right) \sum_{l=0}^{v-r}\left(\begin{array}{c}
v-r \\
l
\end{array}\right) a_{l}^{(c)} E_{v-r-l}
$$

or, equivalently,

$$
\sum_{s=0}^{v}(-1)^{s} \frac{s ! S_{2}(v, s)}{2^{s}}=(2 c) ! 2^{c} \sum_{r=0}^{v}\left(\begin{array}{l}
v \\
r
\end{array}\right) S_{2}\left(r, 2 c ; \frac{1}{2}\right) \sum_{l=0}^{v-r}\left(\begin{array}{c}
v-r \\
l
\end{array}\right) a_{l}^{(c)} E_{v-r-l} .
$$

Remark 2.9. Combining (25) with (24), we have

$$
\sum_{s=0}^{v} S_{2}(v, s) C h_{s}=E_{v}
$$

where $v \in \mathbb{N}_{0}$ (Kim et al., 2013; Simsek, 2019).

\section{CONCLUSION}

Generating functions and $p$-adic integrals have been widely investigated by many mathematicians, physicists, engineers, and other scientists. In particular, the applications of $p$-adic integrals have been frequently used in many different areas. For this reason, here, we gave some interesting formulae for the Fubini type polynomials and numbers by the aid of $p$-adic integrals. These formulae are involved in the Fubini type numbers of higher 
order, the Bernoulli polynomials and numbers, the Euler polynomials and numbers, the Lah numbers, the Stirling type numbers, the combinatorial numbers, and the Hermite polynomials. Consequently, the results of this paper may be usefulness in many areas such as mathematics, engineering and physics.

\section{ACKNOWLEDGEMENT}

The second-named author was supported by the Scientific Research Project Administration of the University of Akdeniz.

\section{CONFLICT OF INTEREST}

The authors declare no conflict of interest.

\section{REFERENCES}

Belbachir, H., Rahmani, M., \& Sury, B. (2011). Sums involving moments of reciprocals of binomial coefficients. Journal of Integer Sequences, 14(6), Article 11.6.6.

Comtet, L. (1974). Advanced Combinatorics: The Art of Finite and Infinite Expansions. Dordrecht and Boston: D. Reidel Publishing.

Kilar, N. (2017). Fubini Type Numbers and Their Generating Functions. MSc Thesis in Mathematics, Akdeniz University, Antalya.

Kilar, N. (2021). Combinatorial sums and identities associated with functional equations of generating functions for Fubini type polynomials. Preprint.

Kilar, N., \& Simsek, Y. (2017). A new family of Fubini numbers and polynomials associated with ApostolBernoulli numbers and polynomials. Journal of the Korean Mathematical Society, 54(5), 1605-1621.

Kilar, N., \& Simsek, Y. (2019a). Some relationships between Fubini type polynomials and other special numbers and polynomials. AIP Conference Proceedings, 2116(100017), 100017.1-100017.4

Kilar, N., \& Simsek, Y. (2019b). Identities and relations for Fubini type numbers and polynomials via generating functions and $p$-adic integral approach. Publications de l'Institut Mathématique, 106(120), 113123.

Kilar, N., \& Simsek, Y. (2021). Formulas and relations of special numbers and polynomials arising from functional equations of generating functions. Montes Taurus Journal of Pure and Applied Mathematics, 3(1), 106-123.

Kim, T. (2002a). q-Volkenborn integration. Russian Journal of Mathematical Physics, 19, 288-299.

Kim, T. (2002b). An invariant $p$-adic integral associated with Daehee numbers. Integral Transforms and Special Functions, 13(1), 65-69.

Kim, T. (2005). A note on $q$-Volkenborn integration. Proceedings of the Jangjeon Mathematical Society, 8(1), $13-17$.

Kim, T. (2007). On the analogs of Euler numbers and polynomials associated with $p$-adic $q$-integral on $\mathbb{Z}_{p}$ at $q=-1$. Journal of Mathematical Analysis and Applications, 331(2), 779-792.

Kim, D. S., \& Kim, T. (2013). Daehee numbers and polynomials. Applied Mathematical Sciences (Ruse), 7 (120), 5969-5976.

Kim, D. S., \& Kim, T. (2018). Some $p$-adic integrals on $\mathbb{Z}_{p}$ associated with trigonometric functions. Russian Journal of Mathematical Physics, 25(3), 300-308.

Kim, D. S., Kim, T., \& Seo, J. (2013). A note on Changhee numbers and polynomials. Advanced Studies in Theoretical Physics, 7, 993-1003.

Kim, T., Kim, D.S., Jang, G.-W., \& Kwon, J. (2018). Symmetric identities for Fubini polynomials. Symmetry, $10,219$. 
OEIS, The On-Line Encyclopedia of Integer Sequences (2021). (Accessed: 14/10/2021) oeis.org/A000670.

Rainville, E. D. (1960). Special Functions. New York: The Macmillan Company.

Riordan, J. (1958). An Introduction to Combinatorial Analysis. New York: John Wiley Sons Inc.

Schikhof, W. H. (1984). Ultrametric Calculus: An Introduction to $p$-adic Analysis. Cambridge Studies in Advanced Mathematics 4, Cambridge: Cambridge University Press.

Simsek, Y. (2013). Generating functions for generalized Stirling type numbers, Array type polynomials, Eulerian type polynomials and their applications. Fixed Point Theory and Applications, 87(2013), 1-28.

Simsek, Y. (2016). Apostol type Daehee numbers and polynomials. Advanced Studies in Contemporary Mathematics, 26, 555-566.

Simsek, Y. (2019). Explicit formulas for $p$-adic integrals: Approach to $p$-adic distributions and some families of special numbers and polynomials. Montes Taurus Journal of Pure and Applied Mathematics, 1(1), 1-76.

Simsek, Y. (2021). Interpolation functions for new classes special numbers and polynomials via applications of $p$-adic integrals and derivative operator. Montes Taurus Journal of Pure and Applied Mathematics, 3(1), $38-61$.

Srivastava, H. M., \& Choi, J. (2012). Zeta and q-Zeta Functions and Associated Series and Integrals. Amsterdam: Elsevier.

Srivastava, H. M., \& Kızılateş, C. (2019). A parametric kind of the Fubini-type polynomials. Revista de la Real Academia de Ciencias Exactas, Físicas y Naturales. Serie A. Matemáticas (RACSAM) 113, 3253-3267. 